\title{
The Radon Transforms on the Generalized Heisenberg Group
}

\author{
Tianwu Liu ${ }^{1,2}$ and Jianxun $\mathrm{He}^{1,2}$ \\ ${ }^{1}$ School of Mathematics and Information Sciences, Guangzhou University, Guangzhou 510006, China \\ ${ }^{2}$ Key Laboratory of Mathematics and Interdisciplinary Sciences of Guangdong Higher Education Institutes, \\ Guangzhou University, Guangzhou 510006, China
}

Correspondence should be addressed to Jianxun He; hejianxun@gzhu.edu.cn

Received 5 September 2013; Accepted 7 October 2013; Published 2 January 2014

Academic Editors: R. Curto and D.-X. Zhou

Copyright (C) 2014 T. Liu and J. He. This is an open access article distributed under the Creative Commons Attribution License, which permits unrestricted use, distribution, and reproduction in any medium, provided the original work is properly cited.

Let $\mathbf{H}_{n}^{a}$ be the generalized Heisenberg group. In this paper, we study the inversion of the Radon transforms on $\mathbf{H}_{n}^{a}$. Several kinds of inversion Radon transform formulas are established. One is obtained from the Euclidean Fourier transform; the other is derived from the differential operator with respect to the center variable $t$. Also by using sub-Laplacian and generalized sub-Laplacian we deduce an inversion formula of the Radon transform on $\mathbf{H}_{n}^{a}$.

\section{Introduction}

In the past decade the research of Radon transform on the Euclidean space $\mathbb{R}^{n}$ has made considerable progress due to its wide applications to partial differential equations, $\mathrm{X}$-ray technology, radio astronomy, and so on. The basic theory and some new developments can be found in [1] by Helgason and the references therein. The combination of Radon transform and wavelet transform has proved to be very useful both on pure mathematics and its applications. Therefore, it is very meaningful to give the inversion formula of the Radon transforms by using various ways. The first result in the area is due to Holschneider who considered the classical Radon transform on the two-dimensional plane (see [2]). Rubin in $[3,4]$ extended the results in [2] to the $k$-dimensional Radon transform on $\mathbb{R}^{n}$ and totally geodesic Radon transforms on the sphere and hyperbolic space. Heisenberg group $\mathbf{H}^{n}$ is a vital Lie group with the underlying $\mathbb{C}^{n} \times \mathbb{R}$. Strichartz [5] discussed the Radon transform on the Heisenberg group. Nessibi and Trimèche [6] obtained an inversion formula of the Radon transform on the Laguerre hypergroup $\mathbf{K}=$ $[0, \infty) \times \mathbb{R}$ by using the generalized wavelet transform. Afterwards, He and Liu studied the analogous problems on the Heisenberg group and Siegel type Lie group (see $[7,8]$ ), and Rubin [9] achieved some new progress of the Radon transform on $\mathbf{H}^{n}$. In [10] the authors gave the definition of generalized Heisenberg group denoted by $\mathbf{H}_{n}^{a}$ and dealt with some problems related to geometric analysis. In this paper, we investigate the inversion formulas of the Radon transform on the generalized Heisenberg group. From the Euclidean Fourier transform and group Fourier transform, we deduce inversion formulas of the Radon transform on $\mathbf{H}_{n}^{a}$ associated with differential operators and generalized sub-Laplacian.

Let $a=\left(a_{1}, a_{2}, \ldots, a_{n}\right)$ be an $n$-dimensional vector, where $a_{i}$ are positive real constants for $i=1,2, \ldots, n$. We can turn $\mathbb{R}^{n} \times \mathbb{R}^{n} \times \mathbb{R}$ into a non-Abelian group by defining the group operation as

$$
\begin{aligned}
& (x, y, t)\left(x^{\prime}, y^{\prime}, t^{\prime}\right) \\
& \quad=\left(x+x^{\prime}, y+y^{\prime}, t+t^{\prime}+\frac{1}{2} \sum_{j=1}^{n} a_{j}\left(x_{j}^{\prime} y_{j}-y_{j}^{\prime} x_{j}\right)\right) .
\end{aligned}
$$

This group is called the generalized Heisenberg group and is denoted by $\mathbf{H}_{n}^{a}$. It is obvious that the generalized Heisenberg group $\mathbf{H}_{n}^{a}$ becomes ordinary Heisenberg group $\mathbf{H}^{n}$ if all $a_{i}=$ 1 for $i=1,2, \ldots, n$. For any $n$-dimensional vectors $a=$ $\left(a_{1}, a_{2}, \ldots, a_{n}\right), b=\left(b_{1}, b_{2}, \ldots, b_{n}\right)$, we define

$$
\begin{gathered}
a * b=\left(a_{1} b_{1}, a_{2} b_{2}, \ldots, a_{n} b_{n}\right), \\
a b=a \cdot b=\sum_{j=1}^{n} a_{j} b_{j} .
\end{gathered}
$$


For $c=\left(c_{1}, c_{2}, \ldots, c_{n}\right), \lambda \in \mathbb{R}$, the following equalities are valid:

(i) $a *(b+c)=a * b+a * c$;

(ii) $(a * b) c=a(b * c)$;

(iii) $(\lambda a) * b=\lambda(a * b)$.

Therefore, (1) can be rewritten as

$$
\begin{aligned}
& (x, y, t)\left(x^{\prime}, y^{\prime}, t^{\prime}\right) \\
& \quad=\left(x+x^{\prime}, y+y^{\prime}, t+t^{\prime}+\frac{1}{2}\left(\left(a * x^{\prime}\right) y-\left(a * y^{\prime}\right) x\right)\right) .
\end{aligned}
$$

Identify $\mathbb{R}^{n} \times \mathbb{R}^{n}$ with $\mathbb{C}^{n}$; the symplectic form $\left(x^{\prime} y-y^{\prime} x\right)$ can be expressed by $\operatorname{Im} z \overline{z^{\prime}}$, where $z=x+i y$ and $z^{\prime}=x^{\prime}+i y^{\prime}$. We can write (1) by

$$
(z, t)\left(z^{\prime}, t^{\prime}\right)=\left(z+z^{\prime}, t+t^{\prime}+\frac{1}{2} \operatorname{Im} \sum_{j=1}^{n} a_{j} z_{j} \overline{z_{j}^{\prime}}\right),
$$

or

$$
(z, t)\left(z^{\prime}, t^{\prime}\right)=\left(z+z^{\prime}, t+t^{\prime}+\frac{1}{2} \operatorname{Im}(a * z) \overline{z^{\prime}}\right) .
$$

In next section, we will introduce some facts of Fourier analysis on $\mathbf{H}_{n}^{a}$, which is useful to get our result.

\section{Fourier Analysis on $\mathbf{H}_{n}^{a}$}

We first state the definition of the Hermite polynomials. Let $\mathbb{Z}^{+}=\{0,1,2, \ldots, n, \ldots\}, k \in \mathbb{Z}^{+}$, and $t \in \mathbb{R}$; the Hermite polynomials are defined by

$$
H_{k}(t)=(-1)^{k}\left(\frac{d^{k}}{d t^{k}}\left\{e^{-t^{2}}\right\} e^{t^{2}}\right) .
$$

The normalized Hermite functions are then defined by

$$
h_{k}(t)=\left(2^{k} \sqrt{\pi} k !\right)^{-(1 / 2)} H_{k}(t) e^{-(1 / 2) t^{2}}
$$

These functions form an orthonormal basis for $L^{2}(\mathbb{R})$. For any fixed $\lambda \in \mathbb{R}^{+}$, it is easy to calculate from (6) that

$$
H_{k}(\sqrt{\lambda} t)=(-1)^{k} \frac{1}{(\sqrt{\lambda})^{k}}\left(\frac{d^{k}}{d t^{k}}\left\{e^{-\lambda t^{2}}\right\} e^{\lambda t^{2}}\right)
$$

then

$$
\begin{aligned}
h_{k}(\sqrt{\lambda} t)= & \left(2^{k} \sqrt{\pi} k !\right)^{-(1 / 2)}(-1)^{k} \frac{1}{(\sqrt{\lambda})^{k}} \\
& \times\left(\frac{d^{k}}{d t^{k}}\left\{e^{-\lambda t^{2}}\right\} e^{\lambda t^{2}}\right) e^{-(1 / 2) \lambda t^{2}} .
\end{aligned}
$$

Therefore, we define

$$
\begin{aligned}
\widetilde{h_{k, \lambda}^{a_{i}}}(t)= & \left(\lambda a_{i}\right)^{1 / 2} h_{k}\left(\sqrt{a_{i} \lambda} t\right) \\
= & \left(\lambda a_{i}\right)^{1 / 2}\left(2^{k} \sqrt{\pi} k !\right)^{-(1 / 2)}(-1)^{k} \\
& \times \frac{1}{\left(\sqrt{a_{i} \lambda}\right)^{k}} \frac{d^{k}}{d t^{k}}\left\{e^{-a_{i} \lambda t^{2}}\right\} e^{\left(a_{i} \lambda / 2\right) t^{2}},
\end{aligned}
$$

which also form the orthonormal basis for $L^{2}(\mathbb{R})$ for any positive constant $a_{i}(i=1,2, \ldots, n)$.

Let $\alpha=\left(\alpha_{1}, \alpha_{2}, \ldots, \alpha_{n}\right) \in\left(\mathbb{Z}^{+}\right)^{n}, x=\left(x_{1}, x_{2}, \ldots, x_{n}\right) \in$ $\mathbb{R}^{n}, a=\left(a_{1}, a_{2}, \ldots, a_{n}\right)$, and $a_{i}>0$ for $i=1,2, \ldots, n$. The higher dimensional Hermite functions denoted by $\Phi_{\alpha}$ can be obtained by taking tensor products:

$$
\Phi_{\alpha}(x)=\prod_{j=1}^{n} h_{a_{j}}\left(x_{j}\right) .
$$

Then the family $\left\{\Phi_{\alpha}: \alpha \in\left(\mathbb{Z}^{+}\right)^{n}\right\}$ is an orthonormal basis for $L^{2}\left(\mathbb{R}^{n}\right)$ (see $\left.[11]\right)$.

We define

$$
\Phi_{\alpha, \lambda}^{a}(x)=\prod_{j=1}^{n} \widetilde{h_{\alpha_{j}, \lambda}^{a_{j}}}\left(x_{j}\right)
$$

then $\left\{\Phi_{\alpha, \lambda}^{a}: \alpha \in\left(\mathbb{Z}^{+}\right)^{n}\right\}$ for any fixed $a$ and $\lambda$ is also the orthonormal basis for $L^{2}\left(\mathbb{R}^{n}\right)$. If $\lambda=1$ and $a_{i}=1$ for $i=$ $1,2, \ldots, n$, then $\Phi_{\alpha}(x)=\Phi_{\alpha, \lambda}^{a}(x)$. And $\left\{\Phi_{\alpha}: \alpha \in\left(\mathbb{Z}^{+}\right)^{n}\right\}$ are eigenfunctions of the Hermite operator $H=-\Delta+|x|^{2}$; that is, $H \Phi_{\alpha}=(2|\alpha|+n) \Phi_{\alpha}$, where $\Delta=\sum_{i=1}^{n} \partial^{2} / \partial x_{i}^{2}$ is Laplacian on $\mathbb{R}^{n}$, and $|\alpha|=\sum_{j=1}^{n} \alpha_{j}$.

For $\lambda \in \mathbb{R} \backslash\{0\}$, let $\pi_{\lambda}(x, y, t)$ be the Schrödinger representation of $\mathbf{H}_{n}^{a}$, which acts on $L^{2}\left(\mathbb{R}^{n}\right)$ by

$$
\pi_{\lambda}(x, y, t) \Phi(\xi)=e^{i \lambda t+i \lambda(a * x) \cdot \xi+(i / 2) \lambda(a * x) \cdot y} \Phi(\xi+y) .
$$

By a direct computation and the law of $\mathbf{H}_{n}^{a}$; we then obtain

$$
\pi_{\lambda}(z, t) \pi_{\lambda}\left(z^{\prime}, t^{\prime}\right)=\pi_{\lambda}\left((z, t)\left(z^{\prime}, t^{\prime}\right)\right),
$$

which indicates that $\pi_{\lambda}$ is unitary. In addition, we deduce that $\pi_{\lambda}$ is irreducible (see [12]).

Suppose that $f \in L^{1}\left(\mathbf{H}_{n}^{a}\right)$, the Fourier transform of $f$ is an operator-valued function acting on $L^{2}\left(\mathbb{R}^{n}\right)$ by

$$
\widehat{f}(\lambda)=\int_{\mathbf{H}_{n}^{a}} f(z, t) \pi_{\lambda}(z, t) d z d t .
$$

If we write $\pi_{\lambda}(z, t)=e^{i \lambda t} \pi_{\lambda}(z)$, where $\pi_{\lambda}(z)=\pi_{\lambda}(z, 0)$, and define

$$
f^{\lambda}(z)=\int_{-\infty}^{+\infty} e^{i \lambda t} f(z, t) d t
$$

then (15) can be rewritten as

$$
\widehat{f}(\lambda)=\int_{\mathbb{R}^{2 n}} f^{\lambda}(z) \pi_{\lambda}(z) d z=\mathbf{W}_{\lambda}\left(f^{\lambda}\right),
$$


where $\mathbf{W}_{\lambda}$ is the Weyl transform. By the same argument of the theory of Weyl transforms on the Heisenberg group we have

$$
\left\|f^{\lambda}\right\|_{L^{2}\left(\mathbb{R}^{2 n}\right)}^{2}=\prod_{i=1}^{n} a_{i}\|\hat{f}(\lambda)\|_{\mathrm{HS}}^{2} .
$$

From this identity we obtain the Plancherel formula

$$
\begin{array}{r}
\|f\|_{L^{2}\left(\mathbf{H}_{n}^{a}\right)}=\left\{\int_{-\infty}^{+\infty}\|\hat{f}(\lambda)\|_{\mathrm{HS}}^{2} d \mu(a, \lambda)\right\}^{1 / 2}, \\
f \in L^{1}\left(\mathbf{H}_{n}^{a}\right) \cap L^{2}\left(\mathbf{H}_{n}^{a}\right),
\end{array}
$$

where $d \mu(a, \lambda)=(2 \pi)^{-n-1}|\lambda|^{n} \prod_{i=1}^{n} a_{i} d \lambda$ and $\|\cdot\|_{\mathrm{HS}}$ is the Hilbert-Schmidt norm of operators. For $f, g \in L^{1}\left(\mathbf{H}_{n}^{a}\right) \cap$ $L^{2}\left(\mathbf{H}_{n}^{a}\right)$, the Parseval formula is

$$
\langle f, g\rangle_{L^{2}\left(\mathbf{H}_{n}^{a}\right)}=\int_{-\infty}^{+\infty} \operatorname{tr}\left(\widehat{g}(\lambda)^{*} \widehat{f}(\lambda)\right) d \mu(a, \lambda),
$$

where $\widehat{g}(\lambda)^{*}$ denotes the adjoint of $\widehat{g}(\lambda)$. For any $(z, t) \in \mathbf{H}_{n}^{a}$, $z=x+i y$, where $x=\left(x_{1}, x_{2}, \ldots, x_{n}\right), y=\left(y_{1}, y_{2}, \ldots, y_{n}\right) \in$ $\mathbb{R}^{n}$. Thus, $(z, t)$ can be written as $(x, y, t) \in \mathbf{H}_{n}^{a}$. We define the left-invariant vector fields $X_{j}, Y_{j}(j=1,2, \ldots, n)$, and $T$ on $\mathbf{H}_{n}^{a}$. These are given by

$$
\begin{array}{cc}
X_{j}=\left(\frac{\partial}{\partial x_{j}}-\frac{1}{2} a_{j} y_{j} \frac{\partial}{\partial t}\right), \quad j=1,2, \ldots, n, \\
Y_{j}=\left(\frac{\partial}{\partial y_{j}}+\frac{1}{2} a_{j} x_{j} \frac{\partial}{\partial t}\right), \quad j=1,2, \ldots, n,
\end{array}
$$

and $T=\partial / \partial t$ (see [10]). The $2 n+1$ vectors fields generate the Lie algebra of the generalized Heisenberg group.

The generalized Heisenberg sub-Laplacian is explicitly given by

$$
\ell=-\sum_{j=1}^{n}\left(X_{j}^{2}+Y_{j}^{2}\right)
$$

Also, a direct computation shows that

$$
\begin{aligned}
\ell= & -\sum_{j=1}^{n}\left(\frac{\partial^{2}}{\partial x_{j}^{2}}+\frac{\partial^{2}}{\partial y_{j}^{2}}\right)-\sum_{j=1}^{n} a_{j}\left(x_{j} \frac{\partial}{\partial y_{j}}-y_{j} \frac{\partial}{\partial x_{j}}\right) \frac{\partial}{\partial t} \\
& -\frac{1}{4} \frac{\partial^{2}}{\partial t^{2}} \sum_{j=1}^{n} a_{j}^{2}\left(x_{j}^{2}+y_{j}^{2}\right) .
\end{aligned}
$$

We know that $\left(-\Delta+|x|^{2}\right) \Phi_{\alpha}=(2|\alpha|+n) \Phi_{\alpha}$ (see [11]). In dimension one, in fact, we have

$$
\left(-\frac{d^{2}}{d x_{1}^{2}}+x_{1}^{2}\right) h_{\alpha_{1}}\left(x_{1}\right)=\left(2 \alpha_{1}+1\right) h_{\alpha_{1}}\left(x_{1}\right) \text {. }
$$

In $n$ dimensions, this equation together with (11) shows that $\Phi_{\alpha}$ is an eigenfunction of the Hermite operators in each variable:

$$
\left(-\frac{d^{2}}{d x_{1}^{2}}+x_{1}^{2}\right) \Phi_{\alpha}(x)=\left(2 \alpha_{1}+1\right) \Phi_{\alpha}(x) .
$$

Therefore, for each $\lambda$, we have

$$
\left(-\frac{d^{2}}{d x_{1}^{2}}+\lambda^{2} x_{1}^{2}\right) \Phi_{\alpha}\left(|\lambda|^{1 / 2} x\right)=|\lambda|\left(2 \alpha_{1}+1\right) \Phi_{\alpha}\left(|\lambda|^{1 / 2} x\right) \text {. }
$$

Let $a_{i}>0$ for $i=1,2, \ldots, n$; then

$$
\begin{aligned}
\sum_{i=1}^{n}\left(-\frac{d^{2}}{d x_{i}^{2}}+\left(a_{i} \lambda\right)^{2} x_{i}^{2}\right) \Phi_{\alpha, \lambda}^{a}(x) & \\
= & \sum_{i=1}^{n}\left(-\frac{d^{2}}{d x_{i}^{2}}+\left(a_{i} \lambda\right)^{2} x_{i}^{2}\right) \\
& \cdot\left(\lambda^{n / 2} \prod_{i=1}^{n} a_{i}^{1 / 2} \prod_{j=1}^{n} h_{\alpha_{j}}\left(\left|a_{j} \lambda\right|^{1 / 2} x_{j}\right)\right) \\
= & |\lambda| \sum_{i=1}^{n} a_{i}\left(2 \alpha_{i}+1\right) \cdot \prod_{i=1}^{n}\left(\left(\lambda a_{i}\right)^{1 / 2} h_{\alpha_{i}}\left(\left|a_{j} \lambda\right|^{1 / 2} x_{i}\right)\right) \\
= & \left(2 \sum_{i=1}^{n} a_{i} \alpha_{i}+\sum_{i=1}^{n} a_{i}\right)|\lambda| \cdot \prod_{j=1}^{n} \widetilde{h_{\alpha_{j}}, \lambda}\left(x_{j}\right) \\
= & (2 a \alpha+|a|)|\lambda| \Phi_{\alpha, \lambda}^{a}(x),
\end{aligned}
$$

where $a \alpha=\sum_{i=1}^{n} a_{i} \alpha_{i}$ and $|a|=\sum_{i=1}^{n} a_{i}$.

Therefore, $\left\{\Phi_{\alpha, \lambda}^{a}: \alpha \in\left(\mathbb{Z}^{+}\right)^{n}\right\}$ are eigenfunctions of operator $\sum_{i=1}^{n}\left(-\left(d^{2} / d x_{i}^{2}\right)+\left(a_{i} \lambda\right)^{2} x_{i}^{2}\right)$ with eigenvalues $(2 a \alpha+$ $|a|)|\lambda|$.

Now we consider the space $\mathbf{H}_{k}$ spanned by $\left\{\Phi_{\alpha, \lambda}^{a}(x)\right.$ : $|\alpha|=k\}$. It is clear that the dimension of $\mathbf{H}_{k}$ is $d_{k}=(n+$ $k-1) ! /(n-1) ! k !$ (see [13]). Then we have

$$
L^{2}\left(\mathbb{R}^{n}\right)=\bigoplus_{k=0}^{\infty} \mathbf{H}_{k}
$$

Let $\mathscr{P}_{k}$ denote the orthogonal projection operator from $L^{2}\left(\mathbb{R}^{n}\right)$ to $\mathbf{H}_{k}$. For $k \in \mathbf{Z}^{+}, \sigma=+$ or-. Let $\widetilde{\mathbf{H}_{k}^{\sigma}}$ be the subspace of $L^{2}\left(\mathbf{H}_{n}^{a}\right)$ such that

$$
\begin{array}{r}
\widetilde{\mathbf{H}_{k}^{+}}=\left\{f \in L^{2}\left(\mathbf{H}_{n}^{a}\right): \widehat{f}(\lambda)=\widehat{f}(\lambda) \mathscr{P}_{k},\right. \\
\left.\mathscr{P}_{k}=0 \text { if } \lambda<0\right\}, \\
\widetilde{\mathbf{H}_{k}^{-}}=\left\{f \in L^{2}\left(\mathbf{H}_{n}^{a}\right): \widehat{f}(\lambda)=\widehat{f}(\lambda) \mathscr{P}_{k},\right. \\
\left.\mathscr{P}_{k}=0 \text { if } \lambda>0\right\} .
\end{array}
$$

Then we have

$$
L^{2}\left(\mathbf{H}_{n}^{a}\right)=\bigoplus_{k=0}^{\infty}\left(\widetilde{\mathbf{H}_{k}^{+}} \oplus \widetilde{\mathbf{H}_{k}^{-}}\right) .
$$

Set $\widetilde{\mathbf{H}_{k}}=\widetilde{\mathbf{H}_{k}^{+}} \oplus \widetilde{\mathbf{H}_{k}^{-}}=\left\{f \in L^{2}\left(\mathbf{H}_{n}^{a}\right): \widehat{f}(\lambda)=\widehat{f}(\lambda) \mathscr{P}_{k}\right\}$; then we can write the above decomposition as

$$
L^{2}\left(\mathbf{H}_{n}^{a}\right)=\bigoplus_{k=0}^{\infty} \widetilde{\mathbf{H}_{k}} .
$$

More details can be found in [14]. 


\section{Inversion of the Radon Transform on $\mathbf{H}_{n}^{a}$}

The Radon transform $R$ for a function $f$ on the generalized Heisenberg group $\mathbf{H}_{n}^{a}$ is defined by

$$
\begin{aligned}
R(f)(z, t) & =\int_{\mathbb{C}^{n}} f\left((z, t)\left(z^{\prime}, 0\right)\right) d z^{\prime} \\
& =\int_{\mathbb{C}^{n}} f\left(z^{\prime}, t+\frac{1}{2} \operatorname{Im} \sum_{j=1}^{n} a_{j} z_{j} \overline{z_{j}^{\prime}}\right) d z^{\prime} \\
& =\int_{\mathbb{R}^{n} \times \mathbb{R}^{n}} f(u, v, t \\
& \left.\quad+\frac{1}{2}((a * y) u-(a * x) v)\right) d u d v
\end{aligned}
$$

where $z^{\prime}=\left(z^{\prime}{ }_{1}, z^{\prime}{ }_{2}, \ldots, z_{n}^{\prime}\right), z^{\prime}=u+i v, z_{j}^{\prime}=u_{j}+i v_{j}, z=x+i y$, and $d z^{\prime}=d u d v$. Clearly, when $a \equiv 1$, that is, $a_{i}=1$ for $i=1,2, \ldots, n$, the above formula is just the Radon transform on the Heisenberg group $\mathbf{H}^{n}$.

Next, we will obtain some inversion formulas for the inverse Radon transform by means of the Euclidean Fourier transform, differential operators, and sub-Laplacian. We first consider the way of the Euclidean Fourier transform. In fact, Strichartz [5] had obtained the inverse Radon transform on $\mathbf{H}^{n}$ by using Euclidean Fourier transform. However, he did not show on which space the formula holds. In this section, we not only find a subspace of $\mathcal{S}\left(\mathbf{H}_{n}^{a}\right)$ on which the Radon transform is a bijection, but also give the inversion Radon transform on $\mathbf{H}_{n}^{a}$, where $\mathcal{S}\left(\mathbf{H}_{n}^{a}\right)$ is the Schwartz space on $\mathbf{H}_{n}^{a}$.

Let $\mathscr{F}_{3}$ denote the Euclidean Fourier transform with respect to the central variable $t$ alone and let $\mathscr{F}$ denote the full Euclidean Fourier transform; that is,

$$
\begin{gathered}
\mathscr{F}_{3}(f)(u, v, \lambda)=\int_{\mathbb{R}} f(u, v, t) e^{i \lambda t} d t \\
\mathscr{F}(f)(x, y, \lambda)=\iint_{\mathbb{R}^{n}} \int_{\mathbb{R}} f(u, v, t) e^{i \lambda t+i u x+i v y} d t d u d v .
\end{gathered}
$$

Because

$$
\begin{aligned}
\mathscr{F}_{3} & (R(f))(x, y, \lambda) \\
= & \int_{\mathbb{R}} R(f)(x, y, t) e^{i \lambda t} d t \\
= & \int_{\mathbb{R}} \int_{\mathbb{R}^{n} \times \mathbb{R}^{n}} f\left(u, v, t+\frac{1}{2}((a * y) u-(a * x) v)\right) \\
= & \times e_{\mathbb{R}^{n} \times \mathbb{R}^{n}} \int_{\mathbb{R}} f(u, v, t) e^{i \lambda t} e^{-(i / 2) \lambda((a * y) u-(a * x) v)} d t d u d v d t \\
= & \int_{\mathbb{R}^{n} \times \mathbb{R}^{n}} \mathscr{F}_{3}(f)(u, v, \lambda) e^{i u(-(\lambda / 2)(a * y))+i v((\lambda / 2)(a * x))} d u d v,
\end{aligned}
$$

we have

$$
\mathscr{F}_{3}(R(f))(x, y, \lambda)=\mathscr{F}(f)\left(-\frac{\lambda}{2}(a * y), \frac{\lambda}{2}(a * x), \lambda\right) .
$$

We define

$$
\begin{array}{r}
\mathcal{S}_{*}\left(\mathbf{H}_{n}^{a}\right) \\
=\left\{f(x, y, t) \in \mathcal{S}\left(\mathbf{H}_{n}^{a}\right): \int_{\mathbb{R}} f(x, y, t) t^{k} d t=0\right. \\
\left.\forall x, y \in \mathbb{R}^{n}, k \in \mathbb{Z}^{+}\right\} \\
\mathcal{S}^{*}\left(\mathbf{H}_{n}^{a}\right)=\left\{f(x, y, t) \in \mathcal{S}\left(\mathbf{H}_{n}^{a}\right):\left.\frac{\partial^{k}}{\partial t^{k}} f(x, y, t)\right|_{t=0}\right. \\
\left.\forall x, y \in \mathbb{R}^{n}, k \in \mathbb{Z}^{+}\right\} .
\end{array}
$$

By argument analogous to [9], we also find that $f \in \mathcal{S}_{*}\left(\mathbf{H}_{n}^{a}\right)$ if and only if $\mathscr{F}(f) \in \mathcal{S}^{*}\left(\mathbf{H}_{n}^{a}\right)$, and $\mathscr{F}_{3}$ is an isomorphism from $\mathcal{S}_{*}\left(\mathbf{H}_{n}^{a}\right)$ onto $\mathcal{S}^{*}\left(\mathbf{H}_{n}^{a}\right)$. The spaces $\mathcal{S}_{*}\left(\mathbf{H}_{n}^{a}\right)$ and $\mathcal{S}^{*}\left(\mathbf{H}_{n}^{a}\right)$ are regarded as Semyanistyi-Lizorkin type spaces that have many applications (see $[6,15])$. We define an operator $\mathscr{J}_{a}$ which is given by

$$
\begin{aligned}
& \mathscr{J}_{a}(f)(x, y, t) \\
& \quad=f\left(-\frac{t}{2} a_{1} y_{1},-\frac{t}{2} a_{2} y_{2}, \ldots,-\frac{t}{2} a_{n} y_{n}, \frac{t}{2} a_{1} x_{1}, \ldots, \frac{t}{2} a_{n} x_{n}, t\right) .
\end{aligned}
$$

It is easy to know that $\mathscr{J}_{a}$ is a bijection on $\mathcal{S}^{*}\left(\mathbf{H}_{n}^{a}\right)$. The inversion of $\mathscr{F}_{a}$ is given by

$$
\begin{aligned}
& \mathscr{F}_{a}^{-1}(f)(x, y, t) \\
& =\left\{\begin{array}{rr}
f\left(-\frac{2 y_{1}}{a_{1} t},-\frac{2 y_{2}}{a_{2} t}, \ldots,-\frac{2 y_{n}}{a_{n} t},\right. \\
\left.\frac{2 x_{1}}{a_{1} t}, \ldots, \frac{2 x_{n}}{a_{n} t}, t\right), & \text { for } t \neq 0 ; \\
0, & \text { for } t=0 .
\end{array}\right.
\end{aligned}
$$

Now (35) reads as

$$
\mathscr{F}_{3}(R(f))(x, y, \lambda)=\mathscr{J}_{a}(\mathscr{F}(f))(x, y, \lambda) .
$$

Therefore, we have an inversion formula of the Radon transform as follows.

Theorem 1. Let $f \in \mathcal{S}_{*}\left(\mathbf{H}_{n}^{a}\right)$. Then one has

$$
R^{-1}(f)=\mathscr{F}^{-1} \mathscr{J}_{a}^{-1} \mathscr{F}_{3}(f) .
$$

We can give another inversion formula of $R$ by using operator $L=\prod_{j=1}^{n} a_{j}((1 / 4 \pi i)(\partial / \partial t))^{n}$. First of all, we give the Fourier transform of Radon transform for a function $f$. 
Lemma 2. Let $f \in L^{2}\left(\mathbf{H}_{n}^{a}\right)$; then

$$
\left(\widehat{R(f)}(\lambda) \Phi_{\alpha}\right)(\eta)=\frac{(-1)^{|\alpha|}|\lambda|^{-n} 2^{n}(2 \pi)^{n}}{\prod_{j=1}^{n} a_{j}}\left(\widehat{f}(\lambda) \Phi_{\alpha}\right)(\eta),
$$

where $\Phi_{\alpha}$ is the orthonormal basis for $L^{2}\left(\mathbb{R}^{n}\right)$.

Proof. Because

$$
\mathscr{F}_{3}(f)(u, v, \lambda)=\int_{\mathbb{R}} f(u, v, t) e^{i \lambda t} d t,
$$

then we have

$$
\begin{aligned}
& \left(\widehat{R(f)}(\lambda) \Phi_{\alpha}\right)(\eta) \\
& =\int_{\mathbf{H}_{n}^{a}} R(f)(x, y, t) e^{i \lambda t+i \lambda(a * x) \eta+(i / 2) \lambda(a * x) y} \\
& \quad \times \Phi_{\alpha}(\eta+y) d x d y d t \\
& =\int_{\mathbb{R}^{n} \times \mathbb{R}^{n}} \mathscr{F}_{3}(f)(u, v, \lambda) \\
& \cdot\left(\int_{\mathbb{R}^{n} \times \mathbb{R}^{n}} e^{i \lambda(a * x) \eta+(i / 2) \lambda(a * x) y-(i / 2) \lambda[(a * y) u-(a * x) v]}\right. \\
& \left.\times \Phi_{\alpha}(\eta+y) d x d y\right) d u d v .
\end{aligned}
$$

Let $\widehat{\Phi_{\alpha}}$ denote the ordinary Fourier transform of $\Phi_{\alpha}$ on $\mathbb{R}^{n}$; then we have

$$
\begin{aligned}
& \int_{\mathbb{R}^{n} \times \mathbb{R}^{n}} e^{i \lambda(a * x) \eta+(i / 2) \lambda(a * x) y-(i / 2) \lambda[(a * y) u-(a * x) v]} \\
& \times \Phi_{\alpha}(\eta+y) d x d y \\
& =\int_{\mathbb{R}^{n}} e^{(i / 2) \lambda(a * u) \eta+(i / 2) \lambda(a * x) \eta+(i / 2) \lambda(a * x) v} \\
& \times\left(\int_{\mathbb{R}^{n}} \Phi_{\alpha}(\eta+y) e^{-(i / 2) \lambda(\eta+y)(a * u-a * x)} d y\right) d x \\
& =\int_{\mathbb{R}^{n}} e^{(i / 2) \lambda(a * u) \eta+(i / 2) \lambda(a * x) \eta+(i / 2) \lambda(a * x) v} \\
& \times \widehat{\Phi_{\alpha}}\left(\frac{\lambda}{2} a *(u-x)\right) d x \\
& =e^{i \lambda(a * u) \eta+(i / 2) \lambda(a * u) v} \\
& \times \int_{\mathbb{R}^{n}} \widehat{\Phi_{\alpha}}\left(\frac{\lambda}{2} a *(u-x)\right) e^{(i / 2) \lambda(a * u-a * x)(-\eta-v)} d x \\
& =\frac{2^{n}|\lambda|^{-n}(2 \pi)^{n}}{\prod_{j=1}^{n} a_{j}} e^{i \lambda(a * u) \eta+(i / 2) \lambda(a * u) v} \Phi_{\alpha}(-v-\eta) .
\end{aligned}
$$

On the other hand, by the recursion formula of Hermite polynomials (see [16]) we can get

$$
\Phi_{\alpha}(-\eta)=(-1)^{|\alpha|} \Phi_{\alpha}(\eta)
$$

so we have

$$
\begin{aligned}
& \left(\widehat{R(f)}(\lambda) \Phi_{\alpha}\right)(\eta) \\
& =\int_{\mathbb{R}^{n} \times \mathbb{R}^{n}} \mathscr{F}_{3} f(u, v, \lambda) \frac{2^{n}|\lambda|^{-n}(2 \pi)^{n}}{\prod_{j=1}^{n} a_{j}} \\
& \quad \times e^{i \lambda(a * u) \eta+(i / 2) \lambda(a * u) v} \Phi_{\alpha}(-v-\eta) d u d v \\
& =\frac{2^{n}|\lambda|^{-n}(2 \pi)^{n}}{\prod_{j=1}^{n} a_{j}} \\
& \quad \times \int_{\mathrm{H}_{n}^{a}} f(u, v, t) e^{i \lambda t+i \lambda(a * u) \eta+(i / 2) \lambda(a * u) v} \\
& \times \Phi_{\alpha}(-v-\eta) d u d v d t \\
& =\frac{(-1)^{|\alpha|}|\lambda|^{-n}(2 \pi)^{n}}{\prod_{j=1}^{n} a_{j}}\left(\widehat{f}(\lambda) \Phi_{\alpha}\right)(\eta) .
\end{aligned}
$$

This completes the proof.

$$
\begin{gathered}
\text { Let } L=\prod_{j=1}^{n} a_{j}((1 / 4 \pi i)(\partial / \partial t))^{n}, f \in \mathcal{S}\left(\mathbf{H}_{n}^{a}\right) ; \text { then } \\
\int_{\mathbb{R}} L(f)(x, y, t) e^{i \lambda t} d t=\frac{(-\lambda)^{n}}{(4 \pi)^{n}} \prod_{j=1}^{n} a_{j} \mathscr{F}_{3}(f)(x, y, \lambda) .
\end{gathered}
$$

Furthermore,

$$
\widehat{L(f)}(\lambda)=\frac{(-\lambda)^{n}}{(4 \pi)^{n}} \prod_{j=1}^{n} a_{j} \widehat{f}(\lambda) .
$$

By (41), we have

$$
\begin{aligned}
& \left(\widehat{\operatorname{LR}(f)}(\lambda) \Phi_{\alpha}\right)(\eta) \\
& \quad= \begin{cases}(-1)^{n+|\alpha|}\left(\widehat{f}(\lambda) \Phi_{\alpha}\right)(\eta), & \text { if } \lambda>0 ; \\
(-1)^{|\alpha|}\left(\hat{f}(\lambda) \Phi_{\alpha}\right)(\eta), & \text { if } \lambda<0 .\end{cases}
\end{aligned}
$$

We can verify that $\widehat{(L R)^{2}(f)}(\lambda)=\widehat{f}$ and $R^{-1}=L R L$.

However, we know that $R(f)$ may not belong to $L^{2}\left(\mathbf{H}_{n}^{a}\right)$ for a function $f \in L^{2}\left(\mathbf{H}_{n}^{a}\right)$. We naturally hope to find a space on which the Radon transform is a bijection. Suppose that $\Omega$ is a subspace of $L^{2}\left(\mathbf{H}_{n}^{a}\right)$ such that the Radon transform $R$ is a bijection. That is, if $f \in \Omega$, then for all $j \in \mathbb{Z}, R^{j}(f) \in \Omega$. From Lemma 2 and the Plancherel formula (19), we have

$$
\left\|R^{j}(f)\right\|_{L^{2}\left(\mathbf{H}_{n}^{a}\right)}^{2}=\frac{(2 \pi)^{2 j n} 4^{j n}}{\prod_{i=1}^{n} a_{i}^{2 j}} \int_{\mathbf{H}_{n}^{a}}\|\hat{f}(\lambda)\|_{\mathrm{HS}}^{2}|\lambda|^{-2 j n} d \mu(a, \lambda) .
$$

Define the subspace $R\left(\mathbf{H}_{n}^{a}\right)$ of $L^{2}\left(\mathbf{H}_{n}^{a}\right)$ by

$$
\begin{aligned}
& R\left(\mathbf{H}_{n}^{a}\right)=\left\{f \in \mathcal{S}\left(\mathbf{H}_{n}^{a}\right):\right. \int_{\mathbb{R}}\|\widehat{f}(\lambda)\|_{\mathrm{HS}}^{2}|\lambda|^{2 j n} d \mu(a, \lambda)<+\infty, \\
&\forall j \in \mathbb{Z}\} .
\end{aligned}
$$


Obviously, if $f_{1}, f_{2} \in R\left(\mathbf{H}_{n}^{a}\right), f_{1} \neq f_{2}$, then $R\left(f_{1}\right) \neq R\left(f_{2}\right)$. Furthermore, for any $g \in R\left(\mathbf{H}_{n}^{a}\right)$, we can find that $f \in R\left(\mathbf{H}_{n}^{a}\right)$, such that $R(f)=g$. In fact, we take $f$ satisfying $\widehat{f}(\lambda)=$ $\prod_{j=1}^{n} a_{j} \cdot(-1)^{|\alpha|}|\lambda|^{n} 2^{-n}(2 \pi)^{-n} \widehat{g}(\lambda)$. Since

$$
\begin{aligned}
\int_{\mathbb{R}}\|\widehat{g}(\lambda)\|_{\mathrm{HS}}^{2}|\lambda|^{2 j n} d \mu(a, \lambda) \\
\quad=\frac{(4 \pi)^{n}}{\prod_{i=1}^{n} a_{i}^{2}} \int_{\mathbb{R}}\|\widehat{f}(\lambda)\|_{\mathrm{HS}}^{2}|\lambda|^{2(j-1) n} d \mu(a, \lambda),
\end{aligned}
$$

we can see that $f \in R\left(\mathbf{H}_{n}^{a}\right)$. This is to say that the Radon transform is a bijection from $R\left(\mathbf{H}_{n}^{a}\right)$ onto itself.

From the above discussion, we have the following.

Theorem 3. Let $f \in R\left(\mathbf{H}_{n}^{a}\right)$; then

$$
R^{-1}(f)=L R L(f) .
$$

Next, we will give another inversion formula associated with the generalized Heisenberg group sub-Laplacian. In fact, a direct calculation shows that

$$
\begin{gathered}
\pi_{\lambda}\left(X_{j}\right) \varphi(\xi)=i \lambda a_{j} \xi_{j} \varphi(\xi), \\
\pi_{\lambda}\left(Y_{j}\right) \varphi(\xi)=\frac{\partial}{\partial \xi_{j}} \varphi(\xi)
\end{gathered}
$$

and consequently

$$
\pi_{\lambda}(\ell)=-\Delta+\lambda^{2}|a * \xi|^{2}
$$

is the scaled Hermite operator, where $X_{j}$ and $Y_{j}$ are the leftinvariant vector fields on $\mathbf{H}_{n}^{a}$.

Because

$$
\sum_{i=1}^{n}\left(-\frac{d^{2}}{d \xi_{i}^{2}}+\left(a_{i} \lambda\right)^{2} \xi_{i}^{2}\right)=-\Delta+\lambda^{2}|a * \xi|^{2}
$$

and $\left\{\Phi_{\alpha, \lambda}^{a}: \alpha \in\left(\mathbb{Z}^{+}\right)^{n}\right\}$ are eigenfunctions of operator $\sum_{i=1}^{n}\left(-\left(d^{2} / d \xi_{i}^{2}\right)+\left(a_{i} \lambda\right)^{2} \xi_{i}^{2}\right)$ with eigenvalues $(2 a \alpha+|a|)|\lambda|$, we have

$$
\pi_{\lambda}(\ell) \Phi_{\alpha, \lambda}^{a}(\xi)=(2 a \alpha+|a|)|\lambda| \Phi_{\alpha, \lambda}^{a}(\xi) .
$$

Set $f \in \widetilde{\mathbf{H}_{k}}$; then we have from [17]

$$
\widehat{\ell f}(\lambda)=(2 a \alpha+|a|)|\lambda| \widehat{f}(\lambda),
$$

where $\sum_{i=1}^{n} \alpha_{i}=k$.

Let $f \in \widetilde{\mathbf{H}_{k}} \cap \mathcal{S}\left(\mathbf{H}_{n}^{a}\right)$; then we can get

$$
\widehat{\ell^{n} f}(\lambda)=(2 a \alpha+|a|)^{n}|\lambda|^{n} \widehat{f}(\lambda) .
$$

Write $\Gamma=\ell^{n}$; by (41), we can deduce

$$
\widehat{\Gamma R f}(\lambda)=\frac{\left(2 \sum_{i=1}^{n} a_{i} \alpha_{i}+n\right)^{n}(-1)^{k} 4^{n} \pi^{n}}{\prod_{j=1}^{n} a_{j}} \widehat{f}(\lambda) .
$$

Consequently, by Plancherel formula we obtain

$$
\begin{aligned}
\left\|(\Gamma R)^{2} f\right\|_{L^{2}\left(\mathbf{H}_{n}^{a}\right)}^{2} & =\int_{-\infty}^{+\infty}\left\|\widehat{(\Gamma R)^{2} f}(\lambda)\right\|_{\mathrm{HS}}^{2} d \mu(a, \lambda) \\
& =\frac{(2 a \alpha+|a|)^{4 n} 4^{4 n} \pi^{4 n}}{\prod_{j=1}^{n} a_{j}^{4}}\|f\|_{L^{2}\left(\mathbf{H}_{n}^{a}\right)}^{2} .
\end{aligned}
$$

Theorem 4. Suppose that $f \in \widetilde{\mathbf{H}_{k}} \cap \mathcal{S}\left(\mathbf{H}_{n}^{a}\right)$; then we have

$$
R^{-1}(f)=\prod_{j=1}^{n} a_{j}^{2} \cdot(2 a \alpha+|a|)^{-2 n}(4 \pi)^{-2 n}(\Gamma R \Gamma)(f),
$$

where $\sum_{i=1}^{n} \alpha_{i}=k$.

We conclude this section by giving the inverse Radon transform with generalized sub-Laplacian.

Let $u_{j}$ be positive constants for $j=1,2, \ldots, n$. We define generalized sub-Laplacian by

$$
\tilde{\ell}=-\sum_{j=1}^{n} u_{j}\left(X_{j}^{2}+Y_{j}^{2}\right)
$$

where $X_{j}$ and $Y_{j}$ are the left-invariant vector fields on $\mathbf{H}_{n}^{a}$. Let $f \in \widetilde{\mathbf{H}_{k}}$; by an analogous computation, we have

$$
\begin{gathered}
\pi_{\lambda}(\widetilde{\ell}) \Phi_{\alpha, \lambda}^{a}(\xi)=|\lambda| \sum_{i=1}^{n} u_{i}\left(2 a_{i} \alpha_{i}+a_{i}\right) \Phi_{\alpha, \lambda}^{a}(\xi), \\
\widehat{\widetilde{\ell} f}(\lambda)=|\lambda| \sum_{i=1}^{n} u_{i}\left(2 a_{i} \alpha_{i}+a_{i}\right) \widehat{f}(\lambda) .
\end{gathered}
$$

If we take $u_{j}=1 / a_{j}$, then

$$
\widehat{\widetilde{\ell} f}(\lambda)=|\lambda|(2 k+n) \widehat{f}(\lambda) .
$$

In this case the Fourier transform of $f$ under the action of generalized sub-Laplacian $\ell$ of $\mathbf{H}_{n}^{a}$ is the same as that of the sub-Laplacian on $\mathbf{H}^{n}$ (see [11]). Write $\widetilde{\Gamma}=\widetilde{\ell}^{n}$; by Lemma 2 , Plancherel formula, and (65), we obtain the theorem below.

Theorem 5. Suppose that $f \in \widetilde{\mathbf{H}_{k}} \cap \mathcal{S}\left(\mathbf{H}_{n}^{a}\right)$. Then we have

$$
R^{-1}(f)=\prod_{j=1}^{n} a_{j}^{2} \cdot\left(\sum_{i=1}^{n} u_{i} a_{i}\left(2 \alpha_{i}+1\right)\right)^{-2 n}(4 \pi)^{-2 n}(\widetilde{\Gamma} R \widetilde{\Gamma})(f),
$$

where $\sum_{i=1}^{n} \alpha_{i}=k$. Especially, when $u_{i}=1 / a_{i}$, we have

$$
R^{-1}(f)=\prod_{j=1}^{n} a_{j}^{2} \cdot(2 k+n)^{-2 n}(4 \pi)^{-2 n}(\widetilde{\Gamma} R \widetilde{\Gamma})(f) .
$$

\section{Conflict of Interests}

The authors declare that there is no conflict of interests regarding the publication of this paper. 


\section{Acknowledgment}

The work for this paper is supported by the National Natural Science Foundation of China (no. 11271091).

\section{References}

[1] S. Helgason, Integral Geometry and Radon Transforms, Springer, New York, NY, USA, 2011.

[2] M. Holschneider, "Inverse Radon transforms through inverse wavelet transforms," Inverse Problems, vol. 7, no. 6, pp. 853-861, 1991.

[3] B. Rubin, "The Calderón reproducing formula, windowed Xray transforms, and Radon transforms in $L^{p}$-spaces," Journal of Fourier Analysis and Applications, vol. 4, no. 2, pp. 175-197, 1998.

[4] B. Rubin, "Fractional calculus and wavelet transforms in integral geometry," Fractional Calculus \& Applied Analysis, vol. 1, no. 2, pp. 193-219, 1998.

[5] R. S. Strichartz, " $L^{p}$ harmonic analysis and Radon transforms on the Heisenberg group," Journal of Functional Analysis, vol. 96, no. 2, pp. 350-406, 1991.

[6] M. M. Nessibi and K. Trimèche, "Inversion of the Radon transform on the Laguerre hypergroup by using generalized wavelets," Journal of Mathematical Analysis and Applications, vol. 208, no. 2, pp. 337-363, 1997.

[7] J. X. He, "An inversion formula of the Radon transform on the Heisenberg group," Canadian Mathematical Bulletin, vol. 47, no. 3, pp. 389-397, 2004.

[8] J. X. He and H. P. Liu, "Admissible wavelets and inverse Radon transform associated with the affine homogenous Siegel domain of type two," Communications in Analysis and Geometry, vol. 15, no. 1, pp. 1-28, 2007.

[9] B. Rubin, "The Radon transform on the Heisenberg group and the transversal Radon transform," Journal of Functional Analysis, vol. 262, no. 1, pp. 234-272, 2012.

[10] O. Calin, D. C. Chang, and P. Greiner, Geometric Analysis on the Heisenberg Group and Its Generalizations, American Mathematical Society, International Press, Providence, RI, USA, 2007.

[11] S. Thangavelu, Harmonic Analysis on the Heisenberg Group, Birkhäuser, Boston, Mass, USA, 1998.

[12] G. B. Folland, Harmonic Analysis in the Phase Space, Princeton University Press, Princeton, NJ, USA, 1989.

[13] E. M. Stein and G. Weiss, Introduction to Fourier Analysis on the Euclidean Spaces, Princeton University Press, Princeton, NJ, USA, 1971.

[14] H. Liu and L. Peng, "Admissible wavelets associated with the Heisenberg group," Pacific Journal of Mathematics, vol. 180, no. 1, pp. 101-123, 1997.

[15] S. G. Samko, A. A. Kilbas, and O. I. Marichev, Fractional Intergrals and Derivatives, Theory and Applications, Gordon and Breach Science Publishers, Yverdon, Switzerland, 1993.

[16] J. B. Seaborn, Hypergeometric Functions and Their Application, vol. 8, Springer, New York, NY, USA, 1991.

[17] D. Geller, "Fourier analysis on the Heisenberg group," Journal of Functional Analysis, vol. 36, no. 2, pp. 205-254, 1980. 


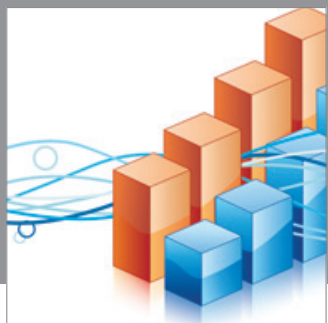

Advances in

Operations Research

mansans

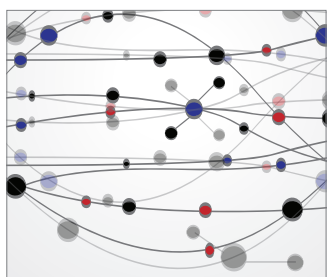

The Scientific World Journal
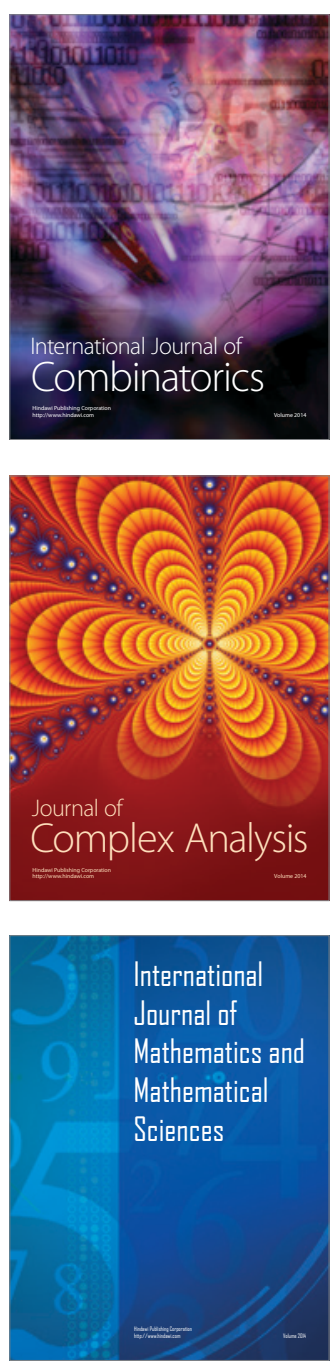
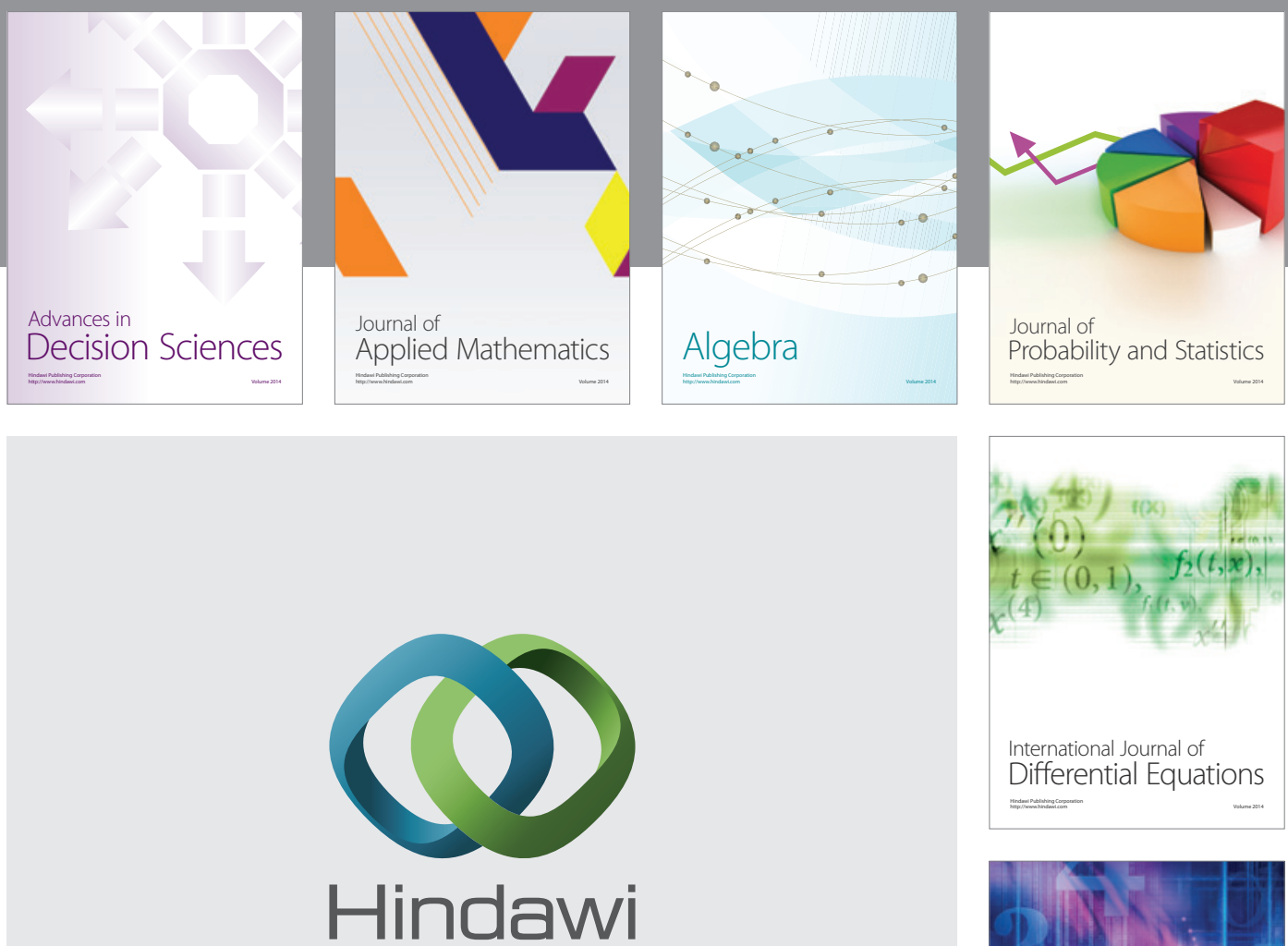

Submit your manuscripts at http://www.hindawi.com
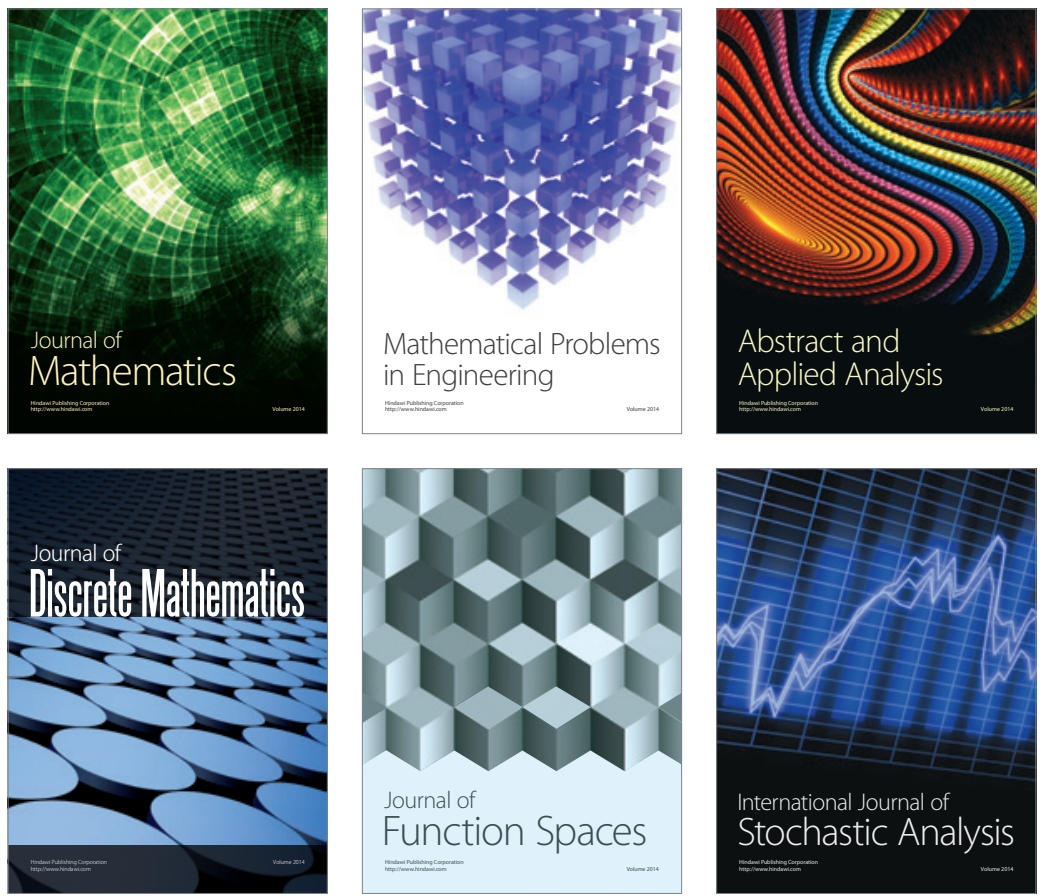

Journal of

Function Spaces

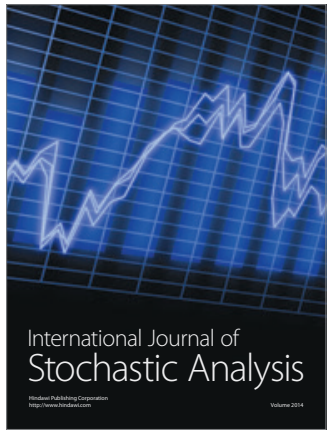

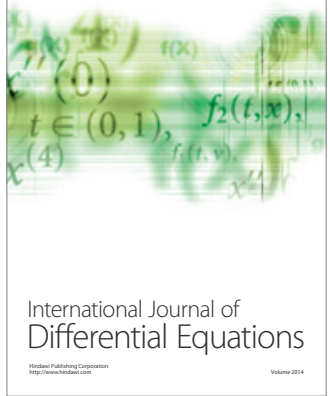
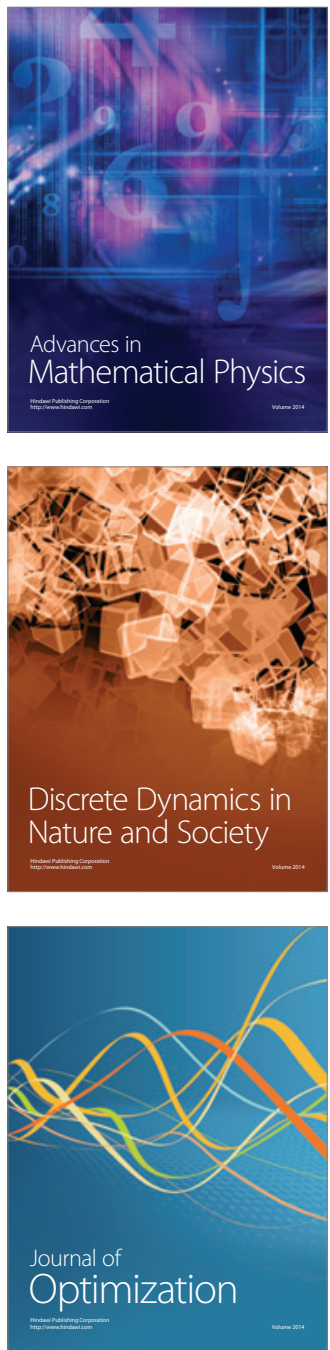